\title{
Observational learning in mice can be prevented by medial prefrontal cortex stimulation and enhanced by nucleus accumbens stimulation
}

\author{
M. Teresa Jurado-Parras, Agnès Gruart, and José M. Delgado-García ${ }^{1}$ \\ Division of Neurosciences, Pablo de Olavide University, 41013-Seville, Spain
}

\begin{abstract}
The neural structures involved in ongoing appetitive and/or observational learning behaviors remain largely unknown. Operant conditioning and observational learning were evoked and recorded in a modified Skinner box provided with an on-line video recording system. Mice improved their acquisition of a simple operant conditioning task by observational learning. Electrical stimulation of the observer's medial prefrontal cortex (mPFC) at a key moment of the demonstration (when the demonstrator presses a lever in order to obtain a reward) cancels out the benefits of observation. In contrast, electrical stimulation of the observer's nucleus accumbens (NAc) enhances observational learning. Ongoing cognitive processes in the demonstrator could also be driven by electrical stimulation of these two structures, preventing the proper execution of the ongoing instrumental task (mPFC) or stopping pellet intake (NAc). Long-term potentiation (LTP) evoked in these two cortical structures did not prevent the acquisition or retrieval process-namely, mPFC and/or NAc stimulation only prevented, or modified, the ongoing behavioral process. The dorsal hippocampus was not involved in either of these two behavioral processes. Thus, both ongoing observational learning and performance of an instrumental task require the active contribution of the mPFC and/or the NAc.
\end{abstract}

[Supplemental material is available for this article.]

Ongoing behaviors are the result of underlying neural processes that determine the sequence of motor activities and, in instrumental conditioning, the relationship between an action and its specific consequences (Balleine and Ostlund 2007). It can be assumed that electrical stimulation of the brain structures putatively involved in those processes affect only behavioral sequences present at the precise moment of the stimulation. For example, it has been reported that electrical stimulation of the medial prefrontal cortex (mPFC) modifies the emotional status (Fuster 2009) or the valuation stage (Kable and Glimcher 2009) of the stimulated animal, but only if this emotion, or valuation need, is present at the very moment of the presentation of the stimulus. Indeed, no observable response was noticed in wild-type mice when stimulated in any of the three cortical areas selected for this study (mPFC, nucleus accumbens [NAc], and hippocampal pyramidal CA1 area) if they were in a resting state or moving around their home cage (Supplemental Video 1).

In order to determine the effects of electrical stimulation of the $\mathrm{mPFC}$ on a well-defined sequence of volitional behaviors, we trained mice in a Skinner box to press a lever to obtain a piece of food delivered from a nearby feeder, using a fixed (1:1) ratio schedule. It has been recently shown that mice can acquire this instrumental conditioning test when lever and feeder are adapted to their sizes (Madroñal et al. 2010). We were then able to stimulate the mPFC at the precise moment of each lever press. We also stimulated the NAc in the same behavioral situation-it has been shown that the NAc is involved in neural circuits related to food intake (Stratford and Kelley 1997; Kelley et al. 2005) and other aspects of feeding behaviors such as food selection according to

\footnotetext{
${ }^{1}$ Corresponding author.

E-mail jmdelgar@upo.es.

Article is online at http://www.learnmem.org/cgi/doi/10.1101/Im.024760.111.
}

their palatability (Halpern et al. 2011). Because the hippocampus has been related with different types of associative (classical eyeblink conditioning) and nonassociative (object recognition, spatial orientation) learning tasks (Gruart et al. 2006; Rossato et al. 2007; Moser et al. 2008; Clarke et al. 2010), for comparative purposes, we stimulated the dorsal hippocampus (pyramidal CA1 area) during the same set of ongoing behaviors.

It is generally accepted that experimentally evoked longterm potentiation (LTP) of cortical circuits is a form of synaptic potentiation sharing many neural processes and properties with learning-dependent changes in synaptic strength (Bliss and Collingridge 1993; Whitlock et al. 2006; Neves et al. 2008). At the least, the similarities between both processes have been convincingly demonstrated at the hippocampal circuits. For example, it has been shown that trace eyeblink conditioning, a form of associative learning, evokes a concomitant change in strength at the hippocampal CA3-CA1 synapse in behaving mice, but this learning can be prevented by the experimental induction of LTP (Gruart et al. 2006). We also checked here if the experimental induction of LTP in the MPFC, NAc, and CA1 sites selected here could prevent the performance of an instrumental learning task. The LTP was evoked by high frequency stimulation (HFS) of the different brain sites (CA3, CA1, mPFC, and NAc) included in this study.

\section{Results}

Electrical stimulation of the mPFC and of the NAc modifies animals' performance of an operant conditioning task in a selective manner

As illustrated in Figure 1, wild-type mice were chronically implanted with stimulating and recording electrodes in the three areas selected for this study: mPFC, NAc, and pyramidal CA1 (see 
A
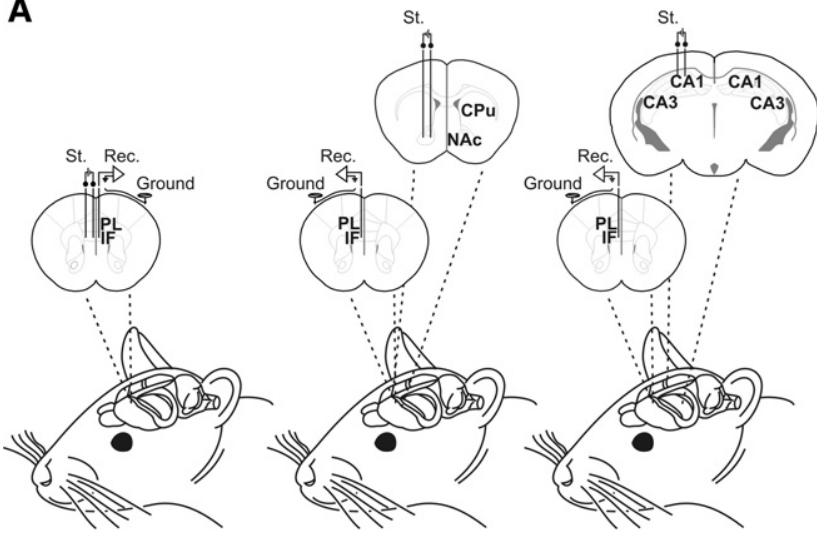

B

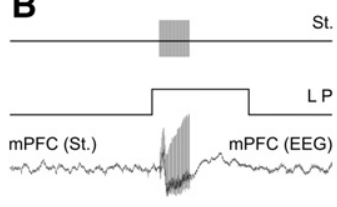

C (1)
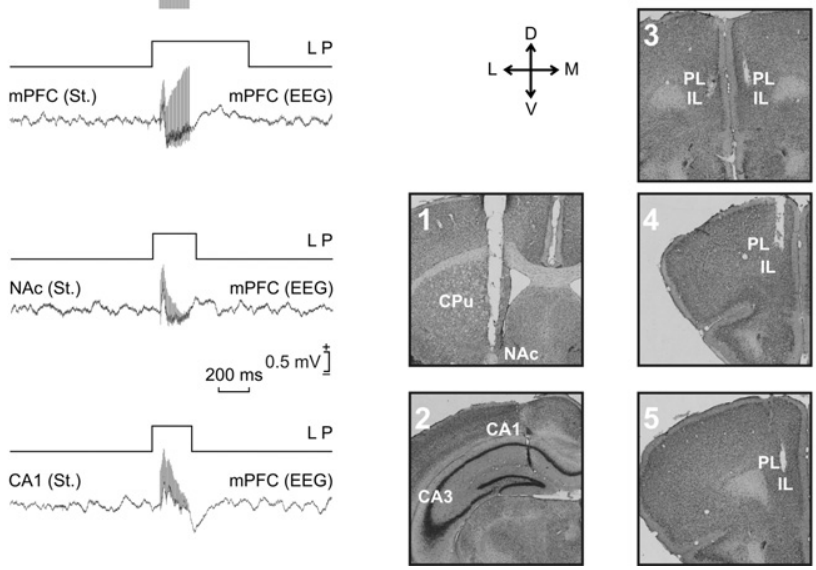

Figure 1. Experimental design. (A) Animals were implanted with bipolar stimulating electrodes in the left $\mathrm{MPFC}$ and recording electrodes in the right $\mathrm{mPFC}$ (left diagram), with stimulating electrodes in the left NAc and recording electrodes in the left mPFC (middle diagram), or stimulating electrodes in the left hippocampal CA1 area and recording electrodes in the left mPFC (right diagram). (B) Representative records collected from the mPFC during lever presses (LP) from animals stimulated in the contralateral MPFC (top), ipsilateral NAc (middle), and ipsilateral hippocampal CA1 area (bottom). Calibration as indicated. (C) Representative photomicrographs of stimulating electrodes implanted in the NAC (1), CA1 area (2), and MPFC (3), and of recording electrodes implanted in the (3-5) mPFC. (CPu) caudate-putamen complex, (IF) infralimbic area of the cortex, (PL) prelimbic area of the cortex, $(D, L, M, V)$ dorsal, lateral, medial, ventral.

Materials and Methods). Following surgery, animals were given operant conditioning in a Skinner box (Fig. 2A). All of the experimental animals ( $n \geq 10$ per experimental group) acquired the operant conditioning task with a fixed-ratio (1:1) schedule (i.e., a food pellet following each lever press) in a mean of $6.5 \pm 0.8$ (SEM) sessions. The criterion was to press the lever a minimum of 20 times/session for two successive 20-min sessions. After reaching the criterion, control mice performed the task consistently, visiting the feeder and getting the pellet after each lever press (Fig. 2A,B), with a performance index close to $1(0.95 \pm$ 0.007) (Fig. 2F). Electrical stimulation of the mPFC (Fig. 1) produced an evident disturbance of this sequential behavior, because, after most $(\approx 57 \%)$ lever presses, the animal did not visit the feeder and did not get the pellet (Figs. 2C, 5C, below; Supplemental Video 2). Furthermore, the time elapsed before visiting the feeder was significantly increased $(P<0.001)$ (Figs. $2 \mathrm{C}, 5 \mathrm{D})$. Although
mPFC-stimulated mice did visit the feeder and got the pellets delivered as effectively as controls did (Fig. 5C), their performance index was $0.73 \pm 0.07$, i.e., significantly $(P<0.001)$ lower than for controls (Fig. 2F). Electrical stimulation of the NAc (Fig. 1) produced a significant $(P \leq 0.05)$ increase in the number of lever presses and a decrease in the number of feeder visits and, most importantly, in the number of eaten pellets (Figs. 2D, 5A,B). These results indicate that this group of animals preferred pressing the lever to visiting the feeder, and that, even having visited the feeder, animals returned to the lever without eating the food reward (Fig. 5C; Supplemental Video 2). In this case, the performance index was significantly $(0.46 \pm 0.07 ; P<0.001)$ (Fig. $2 \mathrm{~F})$ lower than for controls. In contrast, electrical stimulation of the hippocampal pyramidal CA1 area (Fig. 1) did not evoke any behavioral change with respect to values collected from control mice (Figs. 2E,F, $5 \mathrm{~A}-\mathrm{D}$; Supplemental Video 2).

\section{Effects of electrical stimulation of the mPFC and of the NAc on ongoing associative learning tasks were not the result of motor impairments and/or of LTP or LTD evoked at the stimulating sites}

Changes in the ongoing behaviors evoked in mPFC- and NAcstimulated mice were not the result of any motor impairment (Supplemental Video 1), or of long-term potentiation (LTP)- or long-term depression (LTD)-like evoked phenomena (Fig. 3). As already described in alert behaving mice (Gruart et al. 2006; Madroñal et al. 2007), the electrical stimulation of Schaffer collaterals evoked a long-lasting ( $>5 \mathrm{~d}$ ) LTP in the hippocampal CA1 area (Fig. 3A). The HFS of the hippocampal CA1 area and of the mPFC evoked a delayed and long-lasting LTP in mPFC bilaterally (CA1 stimulation) (Fig. 3B,C) or in the contralateral mPFC (mPFC stimulation) (Fig. 3D). This type of delayed LTP has been reported in previous studies (López-Ramos et al. 2011). Interestingly, and as already reported in acute experiments (Robbe et al. 2002; Goto and Grace 2005), HFS of the mPFC evoked a delayed LTD in the ipsilateral NAc (Fig. 3E). In the same way, HFS of the NAc evoked a long-lasting LTD in the mPFC (Fig. 3F). In any case, the presence of experimentally evoked LTP or LTD in the different (CA3, CA1, mPFC, and NAc) brain sites related to the present study did not seem to interfere with the acquisition of the fixed (1:1) ratio schedule (Fig. 3G).

The most parsimonious interpretation of these results was that the timed-locked (i.e., lever-locked) stimulation of mPFC and NAc disturbed specific cognitive processes underlying the expected behavioral sequences. In order to check this hypothesis, we designed another experiment in which the same brain structures were stimulated in animals (i.e., the observers) watching the performance in the Skinner box with a fixed-ratio (1:1) schedule of well-trained animals (i.e., the demonstrators) (Fig. 4A; see Materials and Methods). In this situation, nonstimulated observers reached criterion significantly $(P=0.002)$ before the naive group ( $4.04 \pm 0.4$ sessions vs. $6.5 \pm 0.8$ sessions) (Fig. $4 \mathrm{~B}$ ).

\section{Vicarious learning evoked in alert behaving mice can be prevented by electrical stimulation of the mPFC and improved by electrical stimulation of the NAC}

It has already been reported that both food reward and fear conditioning can be acquired through social observation (Carlier and Jamon 2006; Jeon et al. 2010). Thus, we decided here to study the contribution of mPFC, NAc, and CA1 to observational learning (Supplemental Video 3). Interestingly, mPFC stimulation of the observer when the demonstrator pressed the lever prevented the observer from taking advantage of the observation period, 
A
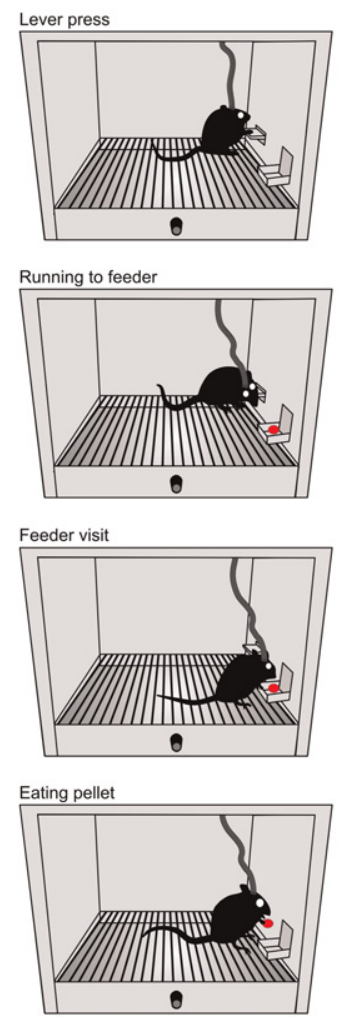

B

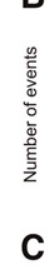

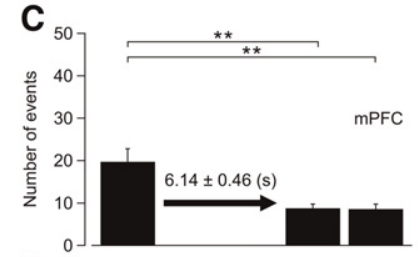

$\mathbf{D}_{50}$

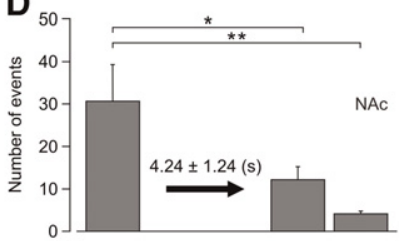

$\mathbf{E}_{50}$

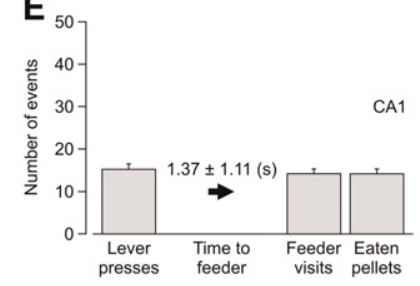

1
$\mathbf{F}$

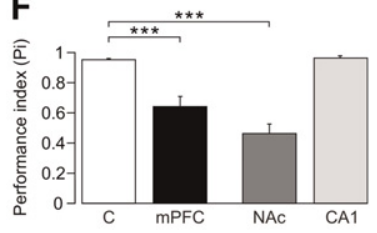

Figure 2. Electrical stimulation of the mPFC and NAc, but not of the hippocampal CA1 area, disturbs the proper performance of an operant conditioning task. (A) Mice were trained in a Skinner box to press a lever to obtain a food pellet with a fixed-ratio (1:1) schedule. (B) Data collected from control mice. The number of lever presses, visits to the feeder, and pellets eaten were quantified, as well as the mean time in seconds (sec) spent from the lever to the feeder (arrow). Sessions lasted for 10 min. (C-E) Effect of electrical stimulation of mPFC (C), NAc (D), and CA1 area (E) on animals' performance. Stimulus consisted of a $200-\mathrm{Hz}$ train of negative-positive $50 \mu \mathrm{sec}$ pulses lasting for $200 \mathrm{msec}$ triggered by each lever press. $(F)$ Performance index $\left(\mathrm{Pi}=\mathrm{N}_{\text {feeder }}+\mathrm{N}_{\text {eat }} / \mathrm{N}_{\text {feeder }}+\mathrm{N}_{\text {lever, }}\right.$ where $\mathrm{N}=$ number of times; see Materials and Methods) for the four experimental groups. Data are presented as mean \pm SEM, $n=9$ animals/group. $(*) P<0.05 ;\left(^{* *}\right) P<0.01 ;\left(^{* * *}\right) P<0.001$.

and they presented acquisition periods similar to those of naive group (6.9 \pm 0.58 sessions vs. $6.5 \pm 0.8$ sessions) (Fig. 4B). In contrast, the timed-locked electrical stimulation of NAc and CA1 did not affect the number of sessions needed to reach criterion (Fig. 4B). Moreover, when the mPFC was stimulated at random (i.e., at times unrelated to the lever presses performed by the demonstrator), stimulated mice reached criterion as control observers did (Fig. 4B). Thus, the disturbing effects of mPFC stimulation seem to be restricted to the sequence of events present at that very time (Curtis and Lee 2010).

Both time-locked (synchronized to the lever press) and random (desynchronized) stimulation of the NAc did not significantly $(P>0.05)$ change the days to criterion and the number of lever presses made during the first conditioning session with respect to values collected from observer animals (Fig. 4B,C). However, the timed-locked electrical stimulation of the NAc produced perceptual enhancement effects-i.e., increased animals' attention to (and movement around) the lever and feeder areas during their first training session in the Skinner box (Fig. 4D). Thus, the activation of the NAc seems to enhance observational learning. The enhancing effects of timed-locked stimulation of the observer's NAc and the disturbance in the cognitive processing of the lever-feeder-food behavioral sequence produced by mPFC stimulation was still noticed in the performance of the different groups of experimental animals across successive training sessions (Fig. 4E).

\section{Discussion}

\section{Role of medial prefrontal cortex circuits in ongoing behaviors}

It is assumed that one of the main roles of the prefrontal cortex is the organization of behavioral actions in the time domain (Fuster 2009). In particular, the medial prefrontal areas seem to be involved in the processing of sensory information related to emotional behavior, including working memories of sensory and motor activities (Fuster and Alexander 1971; Fuster 2009; Hernández et al. 2010). In fact, both lateral and medial prefrontal cortices seem to be involved in observational learning in humans (Burke et al. 2010; Higuchi et al. 2011). As shown here, electrical stimulation of the mPFC seems to disturb ongoing behavioral sequences (Fig. 5) and, probably, the underlying cognitive processes. This conclusion can be deduced from the fact that the electrical stimulation of the observer's mPFC when a demonstrator's performance is being observed disrupts the advantages of the observation, indicating that at this very moment a specific processing of the sensory information collected from the ongoing behavior of the demonstrator is necessary (see Fig. 6).

Previous reports seem to support the above contentions. For example, studies based on the use of repetitive transcraneal magnetic stimulation (rTMS) support a role of prefrontal cortex circuits in imitative learning in humans (see references in Petrosini 
A
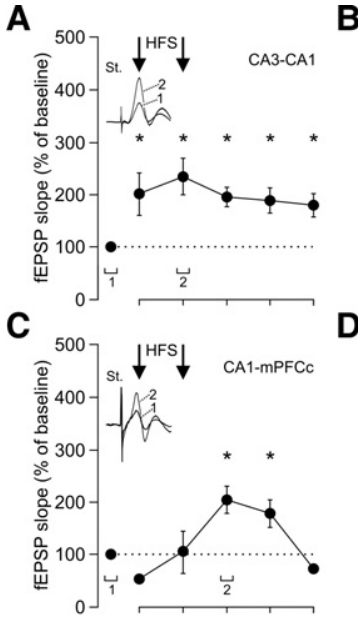

E

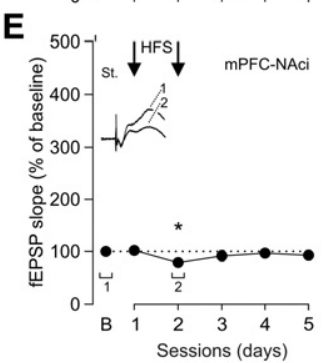

G

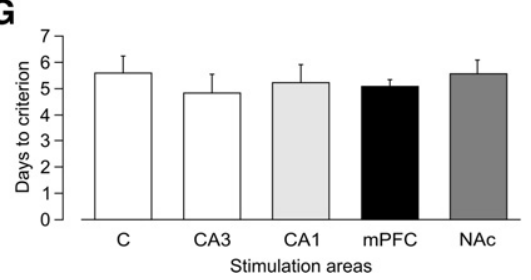

Figure 3. High-frequency stimulation applied to CA3, CA1, mPFC, and NAc did not affect animals' performance in the Skinner box. An attempt was made to evoke long-term potentiation in post-synaptic sites included in this study by HFS of brain centers projecting to them. The HFS protoco consisted of five trains $(200 \mathrm{~Hz}, 100 \mathrm{msec})$ of pulses at a rate of $1 / \mathrm{sec}$. This protocol was presented six times in total, at intervals of $1 \mathrm{~min}$. The HFS protocol was presented on two successive days. In order to obtain a baseline, animals were stimulated every $20 \mathrm{sec}$ for $15 \mathrm{~min}$ in the afferent pathways. After HFS, the same single stimulus was presented at the initial rate ( $3 / \mathrm{min}$ ) for another $30 \mathrm{~min}$. Recording sessions were repeated on three additional days (30 min each). $(A-F)$ Results of HFS of the following synapses: CA3-CA1 (A), CA1-mPFC(i) (B), CA1-mPFC(c) (C), mPFC-mPFC(c) $(D), \operatorname{mPFC}-\mathrm{NAc}(\mathrm{i})(E)$, and NAc-mPFC $(\mathrm{i})(F)$. Both LTP- $(A-D)$ and LTD-like states $(E-F)$ were evoked by the HFS protocol presented here. Averaged $(n=5)$ records collected at the indicated times $(1,2)$ are illustrated for fEPSPs recorded at each synapse. Calibrations in $F$ are also for $A-E$. (G) Days spent by the six stimulated groups included in this study to reach criterion ( $n=20$ lever presses and 20 food rewards during two successive 20-min sessions) as compared with a control group. The operant conditioning sessions were started 30 min after the first HFS session. No significant differences were observed between groups, indicating that LTP or LTD evoked at the selected sites had no effect on the acquisition process. Data are presented as mean \pm SEM, $n \geq 6$ animals/group. (*) $P<0.05$.

2007 and Torriero et al. 2007). Reports indicate that rTMS presented in advance of the observational training interferes with the subsequent performance of already acquired observations. Apparently, both the medial and dorsolateral parts of the prefrontal cortex are involved in imitative learning in humans (Iacoboni 2005). Further studies in humans and monkeys propose that, specifically, the mPFC will be more directly related to a valuation stage (Kable and Glimcher 2009) during the decision process and/ or with the prediction of likely outcomes of the ongoing behaviors (Alexander and Brown 2011). Other studies suggest that the $\mathrm{mPFC}$ is not only involved in the valence but also in the proper timing of the expected outcomes (Forster and Brown 2011). According to the present results, mice seems to use prefrontal cortex processing functions similar to those reported for primates to organize behavioral sequences and to acquire new cognitive and motor abilities by imitation of rewarded behaviors generated by their congeners. Interestingly enough, the availability of a mouse model for the study of prefrontal-executive functions could represent a considerable experimental advantage, merely because of the availability of many genetically manipulated strains able to simulate human cognitive deficits. In this sense, it has been already reported that lesions of the medial prefrontal cortex in rats can reproduce deficits in spatial delayed responses and working memory tasks similar to those presented by humans and monkeys following lesions of the same, as well as more dorsolateral, prefrontal cortex regions (Chudasama 2011).

\section{Differential roles of nucleus accumbens in reward selections and imitative learning}

Results reported here indicate that the NAc could play different roles in ongoing behaviors and cognitive processes depending on the environmental and/or social situations. Thus, during the performance of a fixed (1:1) schedule in a Skinner box, the stimulated animal switched from food reward to electrical-stimulation reward, maintaining, however, most parts of the motor sequence (i.e., even going to the feeder and looking into it). In the case of observational learning, the received (electrical stimulation) reward was apparently associated with the ongoing behaviors of the observed (demonstrator) mouse. In this case, when the stimulated mouse was placed in the Skinner box in the absence of the dividing bars, the animal went directly to the area where the lever is located (Fig. 6).

An interesting result of this study is the switching in rewards, a fact supported by previous findings. It is known that the NAc participates in the central regulation of feeding behaviors (Stratford and Kelley 1997) or even with the palatability of selected nutrients (Halpern et al. 2011). This function is probably accomplished because of NAc projections to the lateral hypothalamus (Stratford and Kelley 1999). In addition, it has been already reported that during sucrose licking in rats, NAc neurons pause and that electrical stimulation of the nucleus (either core or shell) inhibits food consumption (Krause et al. 2010), i.e., the nucleus has been inactive during the reward process, releasing hypothalamic circuits related to feeding behaviors. In contrast, it has also been reported that electrical stimulation of the NAc has a positive rewarding effect (White and Hiroi 1998). According to results collected from the demonstrator's stimulation, it could be assumed that the animal prefers the rewarding effect of lever pressing accompanied by NAc stimulation to collecting a food pellet as a reward (Fig. 2D).

Even so, why does the animal have to visit the lever and feeder areas following the observation of the demonstrator's performance? As suggested above, a possible explanation is obtained when the animal is stimulated in the NAc during lever pressing carried out by the demonstrator. In this situation, social observation predicts that some reward can be gained if the animal moves around the lever and feeder areas (i.e., a sort of local signaling) (Carlier and Jamon 2006), as data illustrated in Figure 4C,D show. In addition, available data suggest that an increase in the activity of NAc neurons (like the one produced by electrical stimulation or by the local administration of dopamine) contributes to an 
A

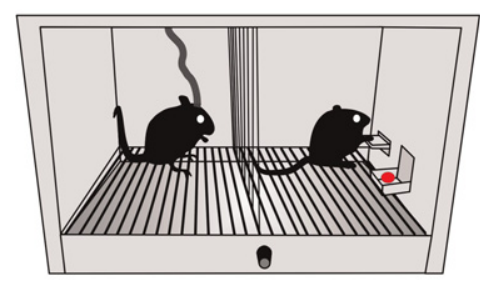

B

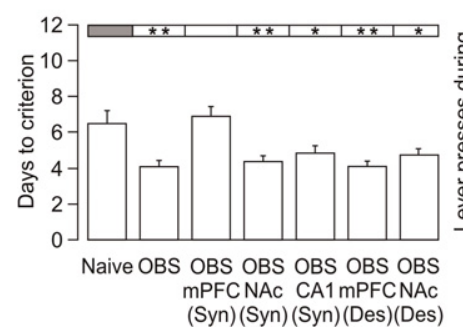

$\mathbf{E}$

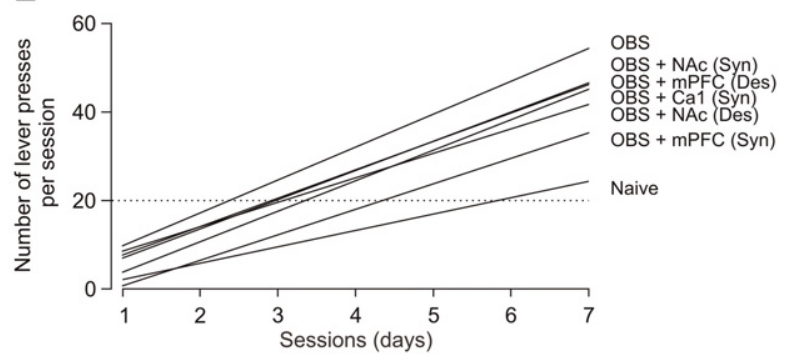

D

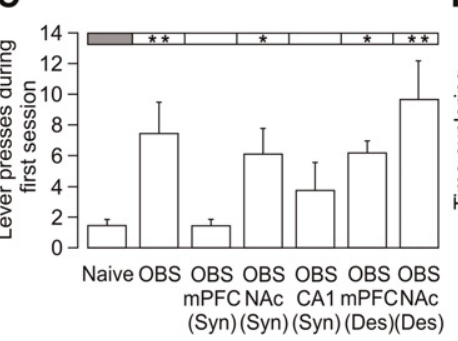

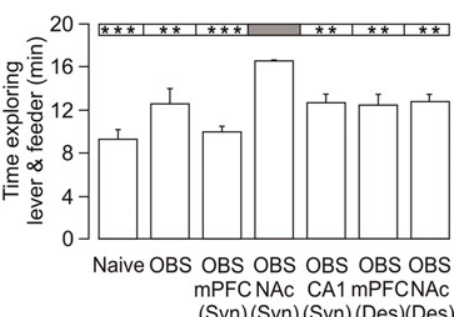

(Syn)(Syn)(Syn)(Des)(Des)

Figure 4. Effects of electrical stimulation of mPFC, NAc, and CA1 on observational learning. ( $A$ ) Observer groups were allowed to watch the performance of a well-trained animal for four 20-min sessions before their own training, while the naive group observed the performance of nontrained animals. Observer animals were stimulated in the mPFC, NAc, or CA1 area each time (and in synchrony [syn]) the demonstrator pressed the lever. Additional observers were stimulated in the mPFC or NAc at random, i.e., desynchronized (des) with respect to lever presses. (B) Time (days) to reach criterion ( $n=20$ lever presses and 20 food rewards during two successive 20-min sessions) for the naive group (Naive), control observers (OBS), and mPFC-, NAc-, or CA1-stimulated observer groups. (C) Mean number of lever presses performed by each group during the first training session. (D) Time spent on the exploration of lever and feeder during the first training session. (E) Evolution of the number of lever presses for all of the groups during seven successive training sessions. The dotted line indicates the criterion level. Data are presented as mean \pm SEM, $n=8$ animals/group. Statistical comparisons are made with respect to the grayed-rectangle group. $\left({ }^{*}\right) P<0.05 ;\left({ }^{* *}\right) P<0.01 ;\left({ }^{* * *}\right) P<0.001$.

increase in the attention to relevant environmental cues (Berridge and Robinson 1998; Wyvell and Berridge 2000, 2001; Nicola et al. 2004). In conclusion, the increased activity of NAc neurons produced by its electrical stimulation could make the animal more attentive to the salience of available environmental cues, discarding already available rewards and looking for new ones, not necessarily related with feeding behaviors.

\section{The dorsal hippocampus is not involved in the performance of already learned sequences of behaviors or in imitative learning}

Our results indicate that the electrical stimulation of the dorsal hippocampus did not affect the (already acquired) behavioral sequence (from lever press to food intake) studied here (Fig. 6). In addition, hippocampal circuits do not seem to be involved in learning by imitation. According to the present information and in agreement with previous reports, the role of the hippocampus is more related to the acquisition of other types of associative learning, such as the classical conditioning of eyelid responses (Gruart et al. 2006; Whitlock et al. 2006), object recognition tasks (Rossato et al. 2007; Clarke et al. 2010), and spatial orientation (Moser et al. 2008), than to the acquisition or performance of operant conditioning tasks.

Interestingly enough, the acquisition and storage of those hippocampal-dependent tasks are easily disrupted by the experimental induction of LTP by HFS of selected sites within the hippocampus (Gruart et al. 2006; Whitlock et al. 2006; Clarke et al. 2010). In the studies reported here, LTP evoked in pathways arriving and/or leaving prefrontal circuits have no noticeable effect on an animal's performance during the fixed-ratio schedule in the Skinner box. These data suggest that the ongoing neural processes taking place in prefrontal circuits can be performed in the pres- ence of the increased synaptic facilitations evoked by the LTP process, a functional capability not available for hippocampal circuits (Gruart et al. 2006; Neves et al. 2008).

\section{Materials and Methods}

\section{Experimental subjects}

Experiments were performed on $\mathrm{C} 57 \mathrm{Bl} / 6$ adult male mice $(3-5$ mo old; $28-35$ g) obtained from an official supplier (University of Granada Animal House, Granada, Spain). Before surgery, animals were housed in separate cages $(n=5$ per cage). The mice were kept on a 12:12-h light : dark cycle with constant ambient temperature $\left(21^{\circ} \mathrm{C} \pm 1{ }^{\circ} \mathrm{C}\right)$ and humidity $(50 \% \pm 7 \%)$. Food and water were available ad libitum. Electrophysiological and behavioral studies were carried out in accordance with the guidelines of the European Union Council (2003/65/CE) and Spanish regulations (BOE 252/34367-91, 2005) for the use of laboratory animals in chronic experiments. Experiments were also approved by the local Ethics Committee of the Pablo de Olavide University (Seville, Spain).

\section{Surgery}

Animals were anesthetized with $0.8 \%-1.5 \%$ isoflurane, supplied from a calibrated Fluotec 5 (Fluotec-Ohmeda) vaporizer, at a flow rate of $1-2 \mathrm{~L} / \mathrm{min}$ oxygen (AstraZeneca) and delivered via a mouse anesthesia mask (David Kopf Instruments). Mice were implanted with bipolar electrodes in the prelimbic/infralimbic area of the $\mathrm{mPFC}(0.3 \mathrm{~mm}$ lateral and $1.94 \mathrm{~mm}$ anterior to bregma, and $1.5-1.7 \mathrm{~mm}$ from the brain surface (Paxinos and Franklin 2001); in the core of the NAc $(1.34 \mathrm{~mm}$ lateral and $1.25 \mathrm{~mm}$ anterior to bregma, and $3.7 \mathrm{~mm}$ from the brain surface); or in the CA1 pyramidal cell layer $(1.2 \mathrm{~mm}$ lateral and $2.2 \mathrm{~mm}$ posterior to bregma, and 1-1.2 $\mathrm{mm}$ from the brain surface). These electrodes were made from $50-\mu \mathrm{m}$, Teflon-coated tungsten wire (Advent 
A

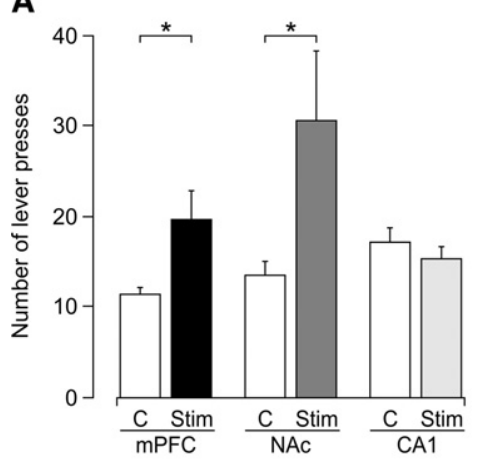

C

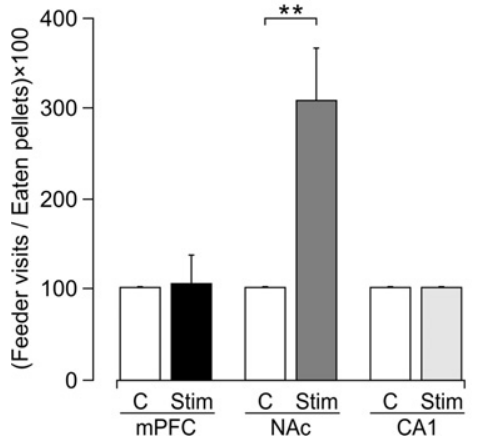

B

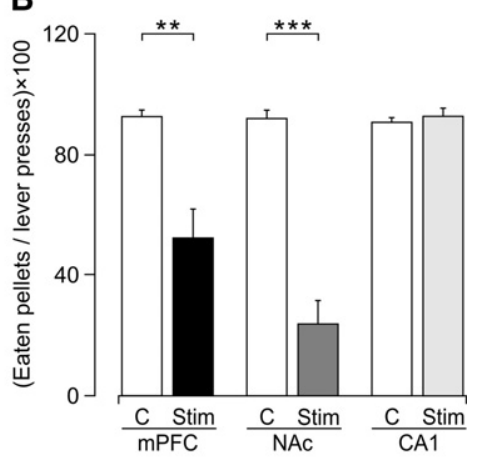

D

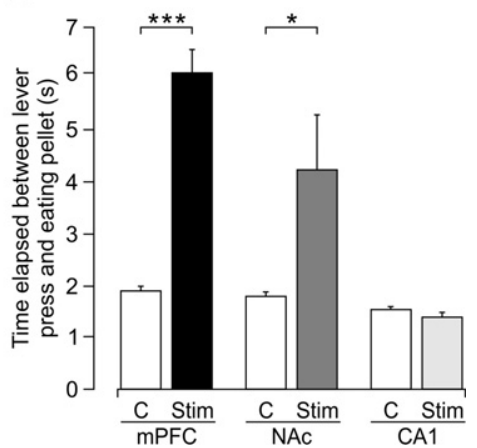

Figure 5. Animals' performance in the Skinner box without or with stimulation in the mPFC, NAc, or CA1 area. The same group of mice were tested in the Skinner box with a fixed-ratio (1:1) schedule in the absence or presence of electrical stimulation in the mPFC, NAc, or CA1 area. Values were collected from 10-min sessions without (C) or with (Stim) stimulation of the indicated brain areas and compared with values collected from the other 10 min of the conditioning session. Illustrated data represent the mean number of lever presses per session $(A)$; the ratio: number of pellets eaten/number of lever presses $(B)$; the ratio: number of visits to the feeder/number of pellets eaten $(C)$; and the time elapsed between pressing the lever and reaching the feeder $(D)$. Quantification indicated that electrical stimulation of the MPFC and NAc significantly increased the number of lever presses and the time taken to visit the feeder and decreased the ratio of pellets eaten/lever presses. Nevertheless, the ratio of feeder visits/ pellets eaten was significantly increased only in the presence of NAc stimulation. No significant differences were observed between sessions with or without CA1 stimulation. Data are presented as mean \pm SEM, $n=9$ animals/group. $\left(^{*}\right) P<0.05 ;\left(^{* *}\right) P<0.01 ;\left({ }^{* *}\right) P<0.001$.

Research) and were used for stimulation or recording purposes as needed. A bare silver wire was affixed to the bone as ground. Implanted wires were soldered to a four-pin socket (RS Amidata) which was fixed to the skull with dental cement (Fig. 1; see Gruart et al. 2006 for details). After surgery, animals were housed in separate cages across the whole experiment.

\section{Operant conditioning procedures}

Training took place in Skinner box modules measuring $12.5 \times$ $13.5 \times 18.5 \mathrm{~cm}$ (MED Associates). Each Skinner box was housed within a sound-attenuating chamber $(90 \times 55 \times 60 \mathrm{~cm})$, which was constantly illuminated (19-W lamp) and exposed to a $45-\mathrm{dB}$ white noise (Cibertec, S.A.). Each Skinner box was equipped with a food dispenser from which pellets (Noyes formula P; $45 \mathrm{mg}$; Sandown Scientific) could be delivered by pressing a lever. Before training, mice were handled daily for $7 \mathrm{~d}$ and food-deprived to $85 \%-75 \%$ of their free-feeding weight.

For operant conditioning, animals were trained to press the lever to receive pellets from the feeder using a fixed-ratio (1:1) schedule. Sessions lasted for $20 \mathrm{~min}$. Animals were maintained on this 1:1 schedule until they reached the selected criterionnamely, until they were able to obtain $\geq 20$ pellets/session for two successive sessions. With these experimental procedures, wild-type mice reached the criterion after 4-7 d of training (Madroñal et al. 2010). Conditioning programs, lever presses, and delivered reinforcements were monitored and recorded by a computer, using a MED-PC program (MED Associates).

Once the criterion was reached, animals were stimulated in the selected (mPFC, NAc, or CA1) brain site every time they pressed the lever. For comparative purposes, stimulations were carried out only during half (i.e., $10 \mathrm{~min}$ ) of the operant conditioning session (Figs. 2, 5). All operant sessions, including brain stimulation and/or electroencephalographic (EEG) and field excitatory postsynaptic potentials (fEPSP) recordings, were recorded with a synchronized video capture system (Sony HDR-SR12E).

For observational learning, the observer was located in a Skinner box in the presence of a well-trained demonstrator (Fig. 4A) for four successive 20-min sessions. For the naive group, the observer was located in the Skinner box in the presence of a nontrained demonstrator. The observer was separated from the demonstrator by a grid similar to the one covering the floor. Observers were stimulated in the selected brain site (mPFC, NAc, or CA1) at a rate of 20-30 stimuli per session either in synchrony with lever presses performed by the demonstrator or generated at random by a computer program. Animals in naive and observer groups were not stimulated. After this pretraining, observers were trained with the above-mentioned operant conditioning task until the expected criterion was reached (Fig. 4).

\section{Recording and stimulating procedures}

For the electrical stimulation of the mPFC, NAc, or CA1 area during operant conditioning, we used a $200-\mathrm{Hz}$ train of pulses (negative-positive for a total of $50 \mu \mathrm{sec})$ lasting for $200 \mathrm{msec}$. The train was triggered by the lever press with a delay $<0.5 \mathrm{msec}$. In order to avoid excessive stimulation of the experimental animal, subsequent lever presses within a 3-sec window did not trigger any stimulus. Stimulus intensities $(0.05-0.25 \mathrm{~mA})$ were selected from previous studies of input/output curves carried out at the synapses included in the LTP study (see below).

For LTP induction in behaving mice, we followed procedures described previously (Gruart et al. 2006; Madroñal et al. 2007). Field EPSP baseline values (Figs. 1, 3) were collected 15 min prior to LTP induction using single $100-\mu$ sec square, biphasic pulses. Pulse intensity was set at $30 \%-40 \%$ of the amount necessary to evoke a maximum fEPSP response $(0.05-0.25 \mathrm{~mA})$-i.e., well below the threshold for evoking a population spike. For LTP induction, animals were presented with two HFS sessions. Each HFS session consisted of five $200-\mathrm{Hz}, 100-\mathrm{msec}$ trains of pulses at a rate of $1 / \mathrm{sec}$ repeated six times, at intervals of $1 \mathrm{~min}$. Thus, a total of 600 pulses were presented during each HFS session. In order to avoid evoking large population spikes and/or the appearance of EEG seizures, the stimulus intensity during HFS was set at the same as that used for generating baseline recordings. After each HFS session, the same single stimuli were presented every $20 \mathrm{sec}$ for $30 \mathrm{~min}$ and for the followings $3 \mathrm{~d}$ (Fig. 3).

\section{Histology}

Once experiments were finished, mice were deeply re-anesthetized (sodium pentobarbital, $50 \mathrm{mg} / \mathrm{kg}$ ) and perfused transcardially 


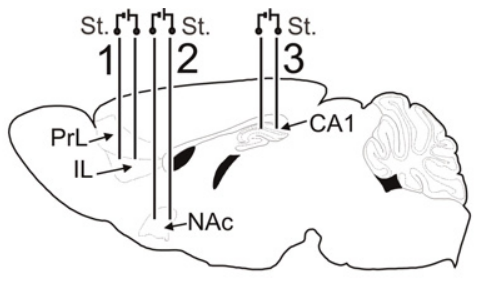

$1 \mathrm{mPFC}$

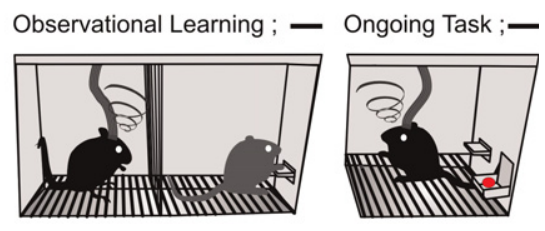

\section{NAc}

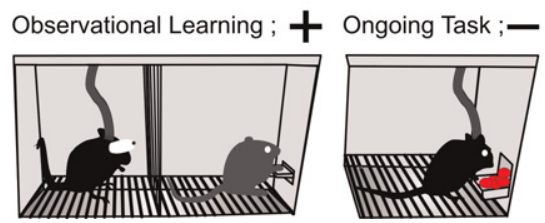

\section{CA1}

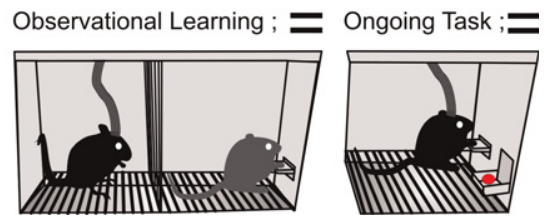

Figure 6. A diagram illustrating the differential effects of $\mathrm{MPFC}, \mathrm{NAc}$, and CA1 stimulation during observational learning and during performance of an operant conditioning task. The diagram at the top left illustrates the different $(1,2,3)$ stimulating sites (mPFC, NAC, and CA1, respectively). (1) The electrical stimulation of the MPFC disturbed both observational learning and the performance of the ongoing task. (2) In the case of NAc stimulation, the animals improved their performance after vicarious learning but switched from food to NAc electrical stimulation reward during the ongoing task. (3) Finally, electrical stimulation of the dorsal hippocampal CA1 area has no effect in any of the tested tasks.

with saline and $4 \%$ phosphate-buffered paraformaldehyde. Their brains were removed, post-fixed overnight at $4^{\circ} \mathrm{C}$, and cryoprotected in $30 \%$ sucrose in PBS. Sections were obtained in a microtome (Leica,) at $50 \mu \mathrm{m}$. Selected sections including the implanted (prefrontal, striatum, and hippocampal) sites were mounted on gelatinized glass slides and stained using the Nissl technique with $0.1 \%$ toluidine blue to determine the location of stimulating and recording electrodes.

\section{Data collection and analysis}

EEG and fEPSP, and 1-volt rectangular pulses corresponding to lever presses, pellet delivery, and brain stimulations were stored digitally on a computer through an analog/digital converter (CED 1401 Plus). Filmed operant conditioning sessions were also stored with the help of the CED 1401 Plus. Data were analyzed off-line for quantification of each animal's performance in the Skinner box, EEG, and fEPSP with the Spike 2 (CED) program. The slope of evoked fEPSPs was computed as the first derivative (volts/sec) of fEPSP recordings (volts). Three to five successive fEPSPs were averaged, and the mean value of the slope during the rise-time period (i.e., the period of the slope between the initial $10 \%$ and the final $10 \%$ of the fEPSP) was determined. Computed results were processed for statistical analysis using the IBM SPSS Statistics 18.0 (IBM).

A performance index was defined as $\mathrm{Pi}=\mathrm{N}_{\text {feeder }}+\mathrm{N}_{\text {eat }} /$ $\mathrm{N}_{\text {feeder }}+\mathrm{N}_{\text {lever, }}$, where $\mathrm{N}=$ number of times the animal visited the feeder $\left(\mathrm{N}_{\text {feeder }}\right)$, ate a pellet $\left(\mathrm{N}_{\text {eat }}\right)$, or pressed the lever $\left(\mathrm{N}_{\text {lever }}\right)$. This Pi enables discriminating all the behavioral possibilities observed here following mPFC, NAc, and CA1 stimulations: in the case of $\mathrm{N}_{\text {feeder }}=\mathrm{N}_{\text {eat }}=\mathrm{N}_{\text {lever }}, \mathrm{Pi}=1$; in the case of $\mathrm{N}_{\text {feeder }}=\mathrm{N}_{\text {lever }}, 0.5 \leq \mathrm{Pi}<1$; in the case of $\mathrm{N}_{\text {eat }}=0,0<\mathrm{Pi} \leq 0.5$; and in the case of $\mathrm{N}_{\text {feeder }}=\mathrm{N}_{\text {eat }} \neq 0,0<\mathrm{Pi}<1$.

Data are always represented as the mean \pm SEM. Statistical significance of differences between groups was inferred by oneway ANOVA and ANOVA for repeated measures (data by groups), with a contrast analysis (Dunnett's post-test) for a further study of significant differences. Statistical significance was set at $\alpha=0.05$.

\section{Acknowledgments}

This study was supported by grants from the Spanish Ministry of Science and Innovation (BFU2008-00899 and BFU2008-03390) and the Junta de Andalucía (Spain, BIO122, CVI-02487 and P07-CVI-02686). The research leading to these results also received funding from the European Community's Seventh Framework Program (FP7/2007-2013) under grant agreement no. 201714 (DEVANX). The authors thank Dr. Raudel SánchezCampusano for designing the performance index, José Antonio Santos for help in the experimental setup, and Roger Churchill for his help in manuscript editing.

\section{References}

Alexander WH, Brown JW. 2011. Medial prefrontal cortex as an action-outcome predictor. Nat Neurosci 14: 1338-1344.

Balleine BW, Ostlund SB. 2007. Still at the choice-point: Action selection and initiation in instrumental conditioning. Ann N Y Acad Sci 1104: 147-171.

Berridge KC, Robinson TE. 1998. What is the role of dopamine in reward: Hedonic impact, reward learning, or incentive salience? Brain Res Brain Res Rev 28: 309-369.

Bliss TVP, Collingridge GL. 1993. A synaptic model of memory: Long-term potentiation in the hippocampus. Nature 361: 31-39.

Burke CJ, Tobler PN, Baddeley M, Schultz W. 2010. Neural mechanisms of observational learning. Proc Natl Acad Sci 107: 14431-14436.

Carlier P, Jamon M. 2006. Observational learning in C57BL/6j mice. Behav Brain Res 174: 125-131.

Chudasama Y. 2011. Animal models of prefrontal-executive function. Behav Neurosci 125: 327-343.

Clarke JR, Cammarota M, Gruart A, Izquierdo I, Delgado-García JM. 2010. Plastic modifications induced by object recognition memory processing. Proc Natl Acad Sci 107: 2652-2657.

Curtis CE, Lee D. 2010. Beyond working memory: The role of persistent activity in decision making. Trends Cogn Sci 14: 216-222.

Forster SE, Brown JW. 2011. Medial prefrontal cortex predicts and evaluates the timing of action outcomes. J Neuroimage 55: 253-265.

Fuster JM. 2009. The prefrontal cortex. Elsevier, Amsterdam.

Fuster JM, Alexander GE. 1971. Neuron activity related to short-term memory. Science 173: 681-697.

Goto Y, Grace AA. 2005. Dopamine-dependent interactions between limbic and prefrontal cortical plasticity in the nucleus accumbens: Disruption by cocaine sensitization. Neuron 47: 255-266.

Gruart A, Muñoz MD, Delgado-García JM. 2006. Involvement of the CA3-CA1 synapse in the acquisition of associative learning in behaving mice. J Neurosci 26: 1077-1087.

Halpern CH, Torres N, Hurtig HI, Wolf JA, Stephen J, Oh MI, Williams NN, Dichter MA, Jaggi JL, Caplan AL, et al. 2011. Expanding applications of deep brain stimulation: A potential therapeutic role in obesity and addiction management. Acta Neurochir 153: 2293-2306.

Hernández A, Nácher V, Luna R, Zainos A, Lemus L, Alvarez M, Vázquez Y, Camarillo L, Romo R. 2010. Decoding a perceptual decision process across cortex. Neuron 66: 300-314.

Higuchi S, Holle H, Roberts N, Eickhoff SB, Vogt S. 2011. Imitation and observational learning of hand actions: Prefrontal involvement and connectivity. J Neuroimage 59: 1668-1683.

Iacoboni M. 2005. Neural mechanisms of imitation. Curr Opi Neurobiol 15: $632-637$.

Jeon D, Kim S, Chetana M, Jo D, Ruley HE, Lin SY, Rabah D, Kinet JP, Shin HS. 2010. Observational fear learning involves affective pain system and $\mathrm{Ca}_{\mathrm{v}} 1.2 \mathrm{Ca}^{2+}$ channels in ACC. Nat Neurosci 13: 482-488.

Kable JW, Glimcher PW. 2009. The neurobiology of decision: Consensus and controversy. Neuron 63: 733-745.

Kelley AE, Baldo BA, Pratt WE, Will MJ. 2005. Corticostriatal-hypothalamic circuitry and food motivation: Integration of energy, action, and reward. Physiol Behav 86: 773-795.

Krause M, German PW, Taha SA, Fields HL. 2010. A pause in nucleus accumbens neuron firing is required to initiate and maintain feeding. J Neurosci 30: 4746-4756.

López-Ramos JC, Jurado-Parras MT, Sanfeliu C, Acuña-Castroviejo D, Delgado-García JM. 2011. Learning capabilities and CA1-prefrontal synaptic plasticity in a mice model of accelerated senescence. Neurobiol Aging 33: e13-e26. doi: 10.1016/j.neurobiolaging.2011.04.005. 
Madroñal N, Delgado-García JM, Gruart A. 2007. Differential effects of long-term potentiation evoked at the CA3-CA1 synapse before, during, and after the acquisition of classical eyeblink conditioning in behaving mice. J Neurosci 27: 12139-12146.

Madroñal N, López-Aracil C, Rangel A, del Río JA, Delgado-García JM, Gruart A. 2010. Effect of enriched physical and social environments on motor performance, associative learning, and hippocampal neurogenesis in mice. PLoS One 5: e11130. doi: 10.1371/journal.pone. 00111.

Moser EI, Kropff E, Moser MB. 2008. Place cells, grid cells, and the brain's spatial representation system. Rev Neurosci 31: 69-89.

Neves G, Cooke SF, Bliss TV. 2008. Synaptic plasticity, memory, and the hippocampus: A neural network approach to causality. Nat Rev Neurosci 9: $65-75$.

Nicola SM, Yun IA, Wakabayasi KT, Fields HL. 2004. Cue-evoked firing of nucleus accumbens neurons encodes motivational significance during a discriminative stimulus task. J Neurophysiol 91: 1840-1865.

Paxinos G, Franklin KBJ. 2001. The mouse brain in stereotaxic coordinates. Academic Press, London.

Petrosini L. 2007. "Do what I do" and "Do how I do": Different components of imitative learning are mediated by different neural structures. Neuroscientist 13: $335-348$.

Robbe D, Kopf M, Remaury A, Bockaert J, Manzoni OJ. 2002. Endogenous cannabinoids mediate long-term synaptic depression in the nucleus accumbens. Proc Natl Acad Sci 99: 8384-8388.

Rossato JI, Bevilaqua LR, Myskiw JC, Medina JH, Izquierdo I, Cammarota M. 2007. On the role of hippocampal protein synthesis in the consolidation and reconsolidation of object recognition memory. Learn Mem 14: 36-46.

Stratford TR, Kelley AE. 1997. GABA in the nucleus accumbens shell participates in the central regulation of feeding behavior. J Neurosci 17: $4434-4440$.

Stratford TR, Kelley AE. 1999. Evidence of a functional relationship between the nucleus accumbens shell and lateral hypothalamus subserving the control of feeding behavior. J Neurosci 19: 11040-11048.

Torriero S, Olivieri M, Koch G, Caltagiore C, Petrosini L. 2007. The what and how of observational learning. J Cogn Neurosci 19: 1656-1663.

White NM, Hiroi N. 1998. Preferential localization of self-stimulation sites in striosomes/patches in the rat striatum. Proc Natl Acad Sci 95: 6486-6491.

Whitlock JR, Heynen AJ, Shuler MG, Bear MF. 2006. Learning induces long-term potentiation in the hippocampus. Science 313: 1093-1097.

Wyvell CL, Berridge KC. 2000. Intra-accumbens amphetamine increases the conditioned incentive salience of sucrose reward: Enhancement of reward "wanting" without enhanced "liking" or response reinforcement. J Neurosci 20: 8122-8130.

Wyvell CL, Berridge KC. 2001. Incentive sensitization by previous amphetamine exposure: Increased cue-triggered "wanting" for sucrose reward. J Neurosci 21: 7831-7840.

Received November 3, 2011; accepted in revised form January 18, 2012. 


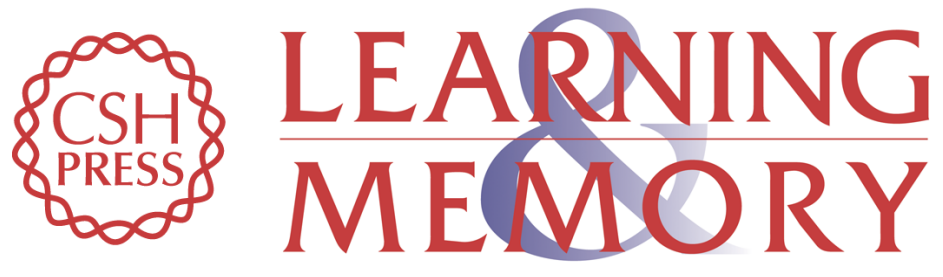

\section{Observational learning in mice can be prevented by medial prefrontal cortex stimulation and enhanced by nucleus accumbens stimulation}

M. Teresa Jurado-Parras, Agnès Gruart and José M. Delgado-García

Learn. Mem. 2012, 19:

Access the most recent version at doi:10.1101//m.024760.111

Supplemental Material

References License

Email Alerting Service
http://learnmem.cshlp.org/content/suppl/2012/02/21/19.3.99.DC1

This article cites 37 articles, 14 of which can be accessed free at: http://learnmem.cshlp.org/content/19/3/99.full.html\#ref-list-1

Receive free email alerts when new articles cite this article - sign up in the box at the top right corner of the article or click here. 